\title{
Existence, uniqueness and stability of fuzzy fractional differential equations with local Lipschitz and linear growth conditions
}

\section{SS Mansouri, M Gachpazan* and O Solaymani Fard}

\section{"Correspondence:}

gachpazan@um.ac.ir

Department of Applied

Mathematics, School of

Mathematical Sciences, Ferdowsi

University of Mashhad, Mashhad, Iran

\begin{abstract}
Fuzzy fractional differential equations (FFDEs) driven by Liu's process are a type of fractional differential equations. In this paper, we intend to provide and prove a novel existence and uniqueness theorem for the solutions of FFDEs under local Lipschitz and linear growth conditions. We also investigate the stability of solutions to FFDEs by a theorem. Finally, some examples are provided.
\end{abstract}

Keywords: credibility theory; fractional derivative; fractional differential equation; fuzzy differential equations; fuzzy Liu's process; existence and uniqueness

\section{Introduction}

A large number of physical processes such as real-life phenomena appear to display fractional-order demeanor that may vary with space or time. The fractional calculus has authorized the operations of differentiation and integration to all fractional order [1-3]. The order may take on all real or imaginary values. Multitude systems modeled with the support of fractional calculus also demonstrate fractional dynamical conduct such as viscoelastic systems $[4,5]$, colored noise [6], boundary layer effects in ducts and electromagnetic waves [7].

In recent decades, the theory of fractional differential equations (FDEs) has enticed many researchers, such as [8-14], who have applied FDEs for acoustic models, thermal systems, signal processing, system identification, robotics and control, etc.

A FDE is a differential equation which includes fractional derivatives. The results of various studies have clearly declared that fractional derivatives seem to arise universally and generally from major mathematical reasons. There are different kinds of fractional derivatives, like Riemann-Liouville and Caputo. For details, one can refer to [12] and [4].

Stochastic fractional differential equations were used to model dynamical systems affected by random noises [15-23].

As already known, the concepts of fuzzy sets have been first investigated by Zadeh via the membership function [24]. The literature concerning the applications of nonlinear FDEs has grown rapidly in the recent years [25-28].

In this paper, we investigate the theory of fuzzy FDEs (FFDEs) in the sense of Liu's process. Liu introduced credibility theory for the first time and presented the concept of credibility measure to facilitate the measurement of fuzzy events [29]. It is worthy to note that

(c) The Author(s) 2017. This article is distributed under the terms of the Creative Commons Attribution 4.0 International License (http://creativecommons.org/licenses/by/4.0/), which permits unrestricted use, distribution, and reproduction in any medium, provided you give appropriate credit to the original author(s) and the source, provide a link to the Creative Commons license, and indicate if changes were made. 
this measurement is a powerful tool for dealing with fuzzy phenomena and is based on normality, monotonicity, self-duality, and maximality axioms.

Also, Liu has proposed the concept of fuzzy process [30-32]. Liu's process is a particular fuzzy process with stationary and independent increment, which is just like a stochastic process described by Brownian motion.

A large number of researches have been recently published on the Liu process and its applications in other sciences such as economics and optimal control [33-38]. Many researchers were inspired by stochastic notions and the Ito process introduced fuzzy differential equations which were driven by Liu's process for better understanding of the fuzzy phenomenon [37, 39-43].

Qin and Li applied fuzzy differential equations, driven by Liu's process, to solve European pricing problems in a fuzzy environment [44].

Regarding the importance of the existence and uniqueness of the solution to fuzzy differential equations driven by Liu's process, You investigated the existence and uniqueness of the solution to the fuzzy differential equations by employing Lipschitz and linear growth conditions [45]. Afterwards, Fei studied the uniqueness of the solution to the fuzzy differential equations driven by Liu's process with non-Lipschitz coefficients [46], but unfortunately, the existence and uniqueness of the solution to the FFDEs driven by Liu's process have not been adequately investigated. Moreover, stability, which is one of the most important issues in differential equations, has not been studied with regard to such equations.

In this paper, weaker conditions are provided in order to guarantee the existence and uniqueness of the solution to the FFDEs, which makes it possible for more functions to be verified in such conditions. Furthermore, stability can also be investigated by considering such conditions.

The basic concepts of credibility theory are first discussed in this paper since they are needed in the later sections. FFDEs driven by Liu's process are taken into consideration in the second section. The existence and uniqueness theorem of the solution with regard to weaker conditions such as the local Lipschitz and Linear growth conditions is proved in the third section, and finally in the last and the most important section, the stability of FFDEs in weaker conditions is elaborated.

\section{Preliminaries}

The emphasis in this section is mainly on introducing some concepts such as credibility measure, credibility space, fuzzy variables, independence of fuzzy variables, expected value, variance, fuzzy process, Liu process, and stopping time.

Suppose that $\Theta$ is a non-empty set and $\mathcal{P}$ is the power set of $\Theta$. Each element of $\kappa$ in $\mathcal{P}$ is called an event. For the purpose of presenting a basic definition of credibility, it is needed to grant a number like $\mathbf{C r}\{\kappa\}$ to each event $\kappa$. In order to make sure the number $\mathbf{C r}\{\kappa\}$ has certain mathematical features that are intuitively expected, these four axioms are accepted [17]:

1. Axiom (normality) $\mathbf{C r}\{\Theta\}=1$.

2. Axiom (monotonicity) $\mathbf{C r}\{\kappa\} \leq \mathbf{C r}\{\beta\}$ whenever $\kappa \subset \beta$.

3. Axiom (self-duality) $\mathbf{C r}\{\kappa\}+\mathbf{C r}\left\{\kappa^{c}\right\}=1$ for any event $\kappa$.

4. Axiom (maximality) $\mathbf{C r}\left\{\mathbf{U}_{i} \kappa_{i}\right\}=\sup _{i} \mathbf{C r}\left\{\kappa_{i}\right\}$ for any events $\left\{\kappa_{i}\right\}$ with

$$
\sup _{i} \operatorname{Cr}\left\{\kappa_{i}\right\}<0.5
$$


Definition 2.1 ([32]) The set function $\mathbf{C r}$ is called a credibility measure if it satisfies the normality, monotonicity, self-duality, and maximality axioms.

A family $\mathcal{P}$ with the above mentioned four properties is called a $\sigma$-algebra. The pair $(\Theta, \mathcal{P})$ is called a measurable space, and each element of $\mathcal{P}$ is consequently called $\mathcal{P}$ measurable sets instead of events.

Definition 2.2 ([47]) The triple $(\Theta, \mathcal{P}, \mathbf{C r})$ is a credibility space if $\Theta$ is a non-empty set, $\mathcal{P}$ the power set of $\Theta$, and $\mathbf{C r}$ a credibility measure.

Let $(\Theta, \mathcal{P}, \mathbf{C r})$ be a credibility space. A filtration is a family $\left\{\mathcal{P}_{t}\right\}_{t \geq 0}$ of increasing sub$\sigma$-algebras of $\mathcal{P}$ (i.e. $\mathcal{P}_{t} \subset \mathcal{P}_{s} \subset \mathcal{P}$ for all $0 \leq t<s<\infty$ ). The filtration is said to be right continuous if $\mathcal{P}_{t}=\bigcap_{s>t} \mathcal{P}_{s}$ for all $t \leq 0$. When the credibility space is complete, the filtration is said to satisfy the usual conditions if it is right continuous and $\mathcal{P}_{0}$ contains all Cr-null sets.

We also define $\mathcal{P}_{\infty}=\sigma\left(U_{t \geq 0} \mathcal{P}_{t}\right)$ (i.e. a $\sigma$-algebra generated by $\left.U_{t \geq 0} \mathcal{P}_{t}\right)$. $\mathcal{P}$-measurable fuzzy variables are denoted by $\mathbf{L}^{p}\left(\Theta, \mathbf{R}^{\mathbf{d}}\right)$, which will be defined later. A process is called $\mathcal{P}$-adapted if for all $t \in[0, t]$ the fuzzy variable $x(t)$ is $\mathcal{P}$-measurable.

Definition 2.3 ([47]) A fuzzy variable is defined as a (measurable) function $\xi:(\Theta, \mathcal{P}$, Cr) $\rightarrow \mathbf{R}$.

Definition 2.4 ([47]) Supposing $\varsigma$ is a fuzzy variable, the expected value of $\varsigma$ is as follows:

$$
\mathbf{E}[s]=\int_{0}^{+\infty} \mathbf{C r}\{s \geq s\} d s-\int_{-\infty}^{0} \operatorname{Cr}\{s \leq s\} d s,
$$

where two integrals are finite.

Definition 2.5 ([47]) The credibility distribution $\eta(x)$ of a fuzzy variable $\varsigma$ is defined by

$$
\eta(w)=\max \{1,2 \mathbf{C r}(\varsigma=w)\}, \quad w \in \mathbf{R} .
$$

Definition 2.6 ([31]) A credibility distribution $\eta(w)$ is regular on condition that it is a continuous and strictly increasing function with respect to $w$ for which $0<\eta(w)<1$, and

$$
\lim _{w \rightarrow-\infty} \eta(w)=0, \quad \lim _{w \rightarrow+\infty} \eta(w)=1 .
$$

Moreover, the inverse function $\eta^{-1}(\alpha)$ can be called the inverse credibility distribution of $\varsigma$.

Definition 2.7 ([47]) Considering $\mathbf{T}$ to be an index set and $(\Theta, \mathcal{P}, \mathbf{C r})$ to be a credibility space, a fuzzy process can be described as a function from $\mathbf{T} \times(\Theta, \mathcal{P}, \mathbf{C r})$ to the set of real numbers.

A fuzzy process is basically a sequence of fuzzy variables indexed by time or space.

Definition 2.8 ([47]) A fuzzy process $\mathbf{C}_{t}$ is a Liu process if the following conditions are met: 
1. $\mathbf{C}_{0}=0$,

2. $\mathbf{C}_{t}$ has stationary and independent increments,

3. every increment $\mathbf{C}_{t+s}-\mathbf{C}_{s}$ is a normally distributed fuzzy variable with expected value $e t$ and variance $\sigma^{2} t^{2}$ whose membership function is

$$
\eta(w)=2\left(1+\exp \left(\frac{\pi|w-e t|}{\sqrt{6} \sigma t}\right)\right)^{-1}, \quad-\infty<w<+\infty
$$

Based on the Liu process, a Liu integral is defined as a fuzzy counterpart of an Ito integral as follows:

Theorem 2.1 ([32]) Let $\mathbf{C}_{t}$ be a standard Liu process, and $f(t, c)$ a continuously differentiable function. Define $w(t)=f\left(t, \mathbf{C}_{t}\right)$. Then we have the following chain rule:

$$
d w(t)=\frac{\partial f}{\partial t}\left(t, \mathbf{C}_{t}\right) d t+\frac{\partial f}{\partial c}\left(t, \mathbf{C}_{t}\right) d \mathbf{C}_{t}
$$

which is called Liu formula.

Let us define a sequence of credibilistic stopping times.

Definition 2.9 ([35]) A fuzzy variable $\tau: \Theta \rightarrow[0, \infty]$ (it may take the value $\infty$ ) is called a $\left\{\mathcal{P}_{t}\right\}$-stopping time (or simply, stopping time) if $\{\theta: \tau(\theta) \leq t\} \in \mathcal{P}_{t}$ for any $t \geq 0$

$$
\begin{cases}\tau_{h}=\inf \{t \geq 0:|w(t)| \geq k\}, & \\ \sigma_{1}=\inf \{t \geq 0: x(w(t)) \geq 2 \varepsilon\}, \\ \sigma_{2 i}=\inf \left\{t \geq \sigma_{2 i-1}: x(w(t)) \leq \varepsilon\right\}, \quad i=1,2, \ldots \\ \sigma_{2 i+1}=\inf \left\{t \geq \sigma_{2 i}: x(w(t)) \geq 2 \varepsilon\right\}, \quad i=1,2, \ldots,\end{cases}
$$

where throughout this paper we set $\inf \phi=\infty$.

Definition 2.10 ([47]) If $W=\left\{W_{t}\right\}_{t \geq 0}$ is a measurable process and $\tau$ is a stopping time, then $\left\{W_{\tau \wedge t}\right\}_{t \geq 0}$ is called a stopped process of $W$.

There are some useful inequalities for fuzzy variables such as the Hölder inequality and the Chebyshev inequality. In this sequence, we introduce generalized inequalities for fuzzy variables.

Theorem 2.2 (Hölder's inequality, [47]) Let $\mathbf{n}$ and $\mathbf{m}$ be two positive real numbers with $\frac{1}{\mathbf{n}}+\frac{1}{\mathbf{m}}=1, \xi$ and $\eta$ be independent fuzzy variables with

$$
\mathbf{E}\left[|\varsigma|^{\mathbf{n}}\right] \leq+\infty \text { and } \mathbf{E}\left[|\rho|^{\mathbf{m}}\right] \leq+\infty
$$

We have

$$
\mathbf{E}[|\zeta \rho|] \leq \sqrt[\mathbf{n}]{\mathbf{E}\left[|\zeta|^{\mathbf{n}}\right]} \sqrt[m]{\mathbf{E}\left[|\rho|^{\mathbf{m}}\right]} .
$$


Theorem 2.3 (Chebychev's inequality) Let $\varsigma: \theta \rightarrow \mathbf{R}^{k}$ be a fuzzy variable such that $\mathbf{E}\left[|\varsigma|^{\mathbf{n}}\right] \leq+\infty$ for some $\mathbf{n}, 0 \leq \mathbf{n} \leq \infty$. Then we have the following Chebychev inequality:

$$
\operatorname{Cr}[|\varsigma| \geq \lambda] \leq \frac{1}{\lambda^{\mathbf{n}}} \mathbf{E}\left[|\varsigma|^{\mathbf{n}}\right] \text { for all } \lambda \geq 0 .
$$

Proof Put $\mathbf{A}=\{x|| \varsigma(x) \mid \geq \lambda\}$. Then

$$
\int_{\theta}|\zeta(x)|^{\mathbf{p}} d \mathbf{C} \mathbf{r}_{x} \geq \int_{A}|\zeta(x)|^{\mathbf{n}} d \mathbf{C} \mathbf{r}_{x} \geq \lambda^{n} \mathbf{C} \mathbf{r}_{A} .
$$

Before ending this section it is essential to introduce some symbols that are used in the next sections.

Notation $1 \mathbf{L}^{\mathbf{n}}\left(\theta, \mathbf{R}^{\mathbf{d}}\right)$ the family of $\mathbf{R}^{\mathbf{d}}$-valued fuzzy variables $\varsigma$ with $\mathbf{E}|\xi|^{\mathbf{P}}<\infty$.

Notation $2 \ell^{\mathbf{P}}\left([a, b], \mathbf{R}^{\mathbf{d}}\right)$ the family of $\mathbf{R}^{\mathbf{d}}$-valued $\mathcal{P}_{t}$-adapted processes $\{h(t)\}_{a \leq t \leq b}$ such that $\int_{a}^{b}|h(t)|^{\mathbf{n}} \mathrm{d} t<\infty$ almost surely.

Notation $3 M^{\mathbf{n}}\left([a, b], \mathbf{R}^{\mathbf{d}}\right)$ the family of processes $\{h(t)\}_{a \leq t \leq b}$ in $\ell^{\mathbf{n}}\left([a, b], \mathbf{R}^{\mathbf{d}}\right)$ such that $\int_{a}^{b}|h(t)|^{\mathbf{n}} d t<\infty$.

Notation $4 \ell^{\mathbf{n}}\left(\mathbf{R}_{+}, \mathbf{R}^{\mathbf{d}}\right)$ the family of processes $\{h(t)\}_{t>0}$ such that, for every $T>0$, $\{h(t)\}_{a \leq t \leq T} \in \ell^{\mathbf{n}}\left([0, T], \mathbf{R}^{\mathbf{d}}\right)$.

Remark 2.1 ([29]) A Liu process $\mathbf{C}$ is Lipschitz continuous, that is, for any given $\theta \in \Theta$, there exists $\mathbf{K}(\theta)>0$ such that for all $t, s \geq 0$

$$
\left|\mathbf{C}_{t_{\theta}}-\mathbf{C}_{s_{\theta}}\right| \leq|t-s|
$$

Lemma 2.1 ([31]) Suppose that $\mathbf{C}_{t}$ is a standard Liu process and $x(t)$ is a fuzzy process on $[a, b]$ with respect to $t$. If $\mathbf{K}(\Theta)>0$ is the Lipschitz constant for path $\mathbf{C}_{t_{\theta}}$ with $\theta \in \Theta$ fixed, then we have

$$
\left|\int_{a}^{b} x(t) d \mathbf{C}_{t}\right| \leq \mathbf{K}(\theta) \int_{a}^{b}|x(t)| d t .
$$

\section{Fuzzy fractional differential equations}

In this section, some basic definitions, notations and lemmas which will be used throughout the paper are provided in order to establish our main results.

Two common notations for the fractional differential operator also need to be introduced, which are Riemann-Liouville and Caputo. See [12] and [4] for more details.

Definition 3.1 ([12]) The Riemann-Liouville fractional derivative of $f$ is defined as

$$
{ }^{R} D^{\alpha} f(t)=\frac{1}{\Gamma(1-\alpha)} \frac{d}{d t} \int_{0}^{t}(t-s)^{-\alpha} f(s) d s,
$$

where $\Gamma(\cdot)$ stands for the gamma function $\Gamma(x)=\int_{0}^{\infty} t^{x-1} e^{-t} d t, \alpha \in(0,1]$, and $t>0$. 
Definition $3.2([4])$ The Caputo derivative of order $\alpha$ for a function $\mathrm{f}$ can be written as

$$
{ }^{C} D^{\alpha} f(t)=\frac{1}{\Gamma(1-\alpha)} \int_{0}^{t}(t-s)^{-\alpha} f^{\prime}(s) d s
$$

where $f^{\prime}(s)$ is the first-order derivative of $f(s)$.

Remark 3.1 The relationship between the Riemann-Liouville derivative and the Caputo derivative can be written as

$$
{ }^{R} D^{\alpha} f(t)={ }^{C} D^{\alpha} f(t)+\frac{t^{-\alpha}}{\Gamma(1-\alpha)} f(0) .
$$

In this study, we consider the Caputo fractional derivative of order $\alpha$, and the initial value problem of fuzzy fractional-order differential equation is given as follows:

$$
\left\{\begin{array}{l}
{ }^{C} D^{\alpha} w(t)=f(w(t), t)+g(w(t), t) \dot{C}(t), \\
w(t)=w_{t}
\end{array}\right.
$$

where the functions $f(w(t), t)$ and $g(w(t), t):[0, T] \times R \rightarrow R$. The term $\dot{C}(t)=\frac{d C}{d t}$ describes a state dependent random noise. $C(t)$ is a standard Liu process defined on a given filtered credibility space $\left(\Theta, \mathcal{P},\left\{\mathcal{P}_{t}\right\}_{t \geq 0}, \mathbf{C r}\right)$ with a normal filtration $\left\{\mathcal{P}_{t}\right\}_{t \geq 0}$, which is an increasing and continuous family of $\sigma$-algebras of $\mathcal{P}$, and contains all of $\Theta$-null sets, and $C(t)$ is $\mathcal{P}$ measurable for each $t>0$. Here, let us recall the definitions of fractional calculus; the fractional integral operator of order $\alpha$ is given as follows:

$$
I^{\alpha} g(t)=\frac{1}{\Gamma(\alpha)} \int_{0}^{t}(t-s)^{\alpha-1} g(s) d s, \quad t>0 .
$$

Applying the integral operator (3.5) to both sides of initial value problem (3.4), the Volterra integral equation is obtained:

$$
w(t)=w_{0}+\frac{1}{\Gamma(\alpha)} \int_{0}^{t}(t-s)^{\alpha-1} f(w(s), s) d s+\frac{1}{\Gamma(\alpha)} \int_{0}^{t}(t-s)^{\alpha-1} g(w(s), s) d C(s),
$$

in which $\alpha \in(0,1)$ and $t>0$.

Lemma 3.1 Let function $K(u, t)$ be locally integrable in $t$ for each fixed $u \in[0, \infty]$ and be continuously non-decreasing in $u$ for each fixed $t \in[0, T]$, for $K(0, t)=0$ and $\gamma>0$. If a non-negative continuous function $z(t)$ satisfies

$$
z(t) \leq \gamma \int_{0}^{t} K(z(s), s) d s, \quad t \in R, z(0)=0
$$

then $z(t)=0$ for all $t \in[0, T]$.

\subsection{Existence and uniqueness of the solution for FFDEs}

In this section, existence and uniqueness of the solution for FFDEs are taken into consideration. It is worth noting that, if FFDE is a mathematical model of a physical problem: 
1. We expect initial conditions of the mathematical model to have a solution.

2. According to initial conditions, we want any mathematical model to have a unique solution.

First, a suitable condition proposes a uniqueness solution to FFDEs.

At this point the following conditions, lemmas, and remarks for the proof of the uniqueness and existence should be elaborated on.

(I) Local Lipschitz condition: There exists a positive constant number $\mathbf{L}$ such that

$$
|p(w(t), t)-p(y(t), t)|+|q(w(t), t)-q(y(t), t)| \leq \mathbf{L}|w(t)-y(t)|
$$

for those $w(t), y(t) \in \mathbf{R}^{n}$.

(II) Linear growth condition: There exists a positive number $\mathbf{L}$ such that

$$
|p(w(t), t)|+|q(w(t), t)| \leq \mathbf{L}(1+|w(t)|) .
$$

Now, we introduce the mapping $\mathbf{A}$ on $\mathbf{C}[0, \mathbf{T}]$ for $W(t) \in \mathbf{C}[0, \mathbf{T}]$ as follows:

$$
\mathbf{A}(w(t))=w_{0}+\frac{1}{\Gamma(\alpha)} \int_{0}^{t}(t-s)^{p-1} f(w(s), s) d s+\frac{1}{\Gamma(\alpha)} \int_{0}^{t}(t-s)^{p-1} g(w(s), s) d C(s),
$$

where $t \in[0, T], \tau \geq 0$.

Lemma 3.2 For any $\tau \in \Theta$, and $w(t, \tau) \in \mathbf{C}([-\tau, \mathbf{R}], \mathbf{R})$ we have $A(w(t, \tau)) \in \mathbf{C}([-\tau, \mathbf{R}], \mathbf{R})$.

Proof Let $s_{1}, s_{2} \in[0, T]$ and $s_{2}>s_{1}>0$. We have

$$
\begin{aligned}
\mid \mathbf{A}(w & \left.\left(s_{2}, \theta\right)\right)-\mathbf{A}\left(w\left(s_{1}, \theta\right)\right) \mid \\
= & \frac{1}{\Gamma(p)} \mid \int_{0}^{s_{1}}\left(s_{2}-s\right)^{p-1} f(w(s, \tau), s) d s+\int_{0}^{s_{1}}\left(s_{2}-s\right)^{p-1} g(w(s, \tau), s) d \mathbf{C}_{s}(\tau) \\
& +\int_{s_{1}}^{s_{2}}\left(s_{2}-s\right)^{p-1} f(w(s, \tau), s) d s+\int_{s_{1}}^{s_{2}}\left(s_{2}-s\right)^{p-1} g(w(s, \tau), s) d \mathbf{C}_{s}(\tau) \\
& -\int_{0}^{s_{1}}\left(s_{1}-s\right)^{p-1} f(w(s, \tau), s) d s+\int_{0}^{s_{1}}\left(s_{1}-s\right)^{p-1} g(w(s, \tau), s) d \mathbf{C}_{s}(\tau) \mid \\
= & \frac{1}{\Gamma(p)} \mid \int_{0}^{s_{1}}\left[\left(s_{2}-s\right)^{p-1}-\left(s_{1}-s\right)^{p-1}\right] f(w(s, \tau), s) d s \\
& +\int_{0}^{s_{1}}\left[\left(s_{2}-s\right)^{p-1}-\left(s_{1}-s\right)^{p-1}\right] g(w(s, \tau), s) d \mathbf{C}_{s}(\tau) \\
& +\int_{s_{1}}^{s_{2}}\left(s_{2}-s\right)^{p-1} f(w(s, \tau), s) d s+\int_{s_{1}}^{s_{2}}\left(s_{2}-s\right)^{p-1} g(x(s, \tau), s) d \mathbf{C}_{s}(\tau) \mid \\
\leq & \frac{1}{\Gamma(p)} \int_{0}^{s_{1}}\left[\left(s_{2}-s\right)^{p-1}-\left(s_{1}-s\right)^{p-1}\right]|f(w(s, \tau), s)| d s \\
& +\left|\int_{0}^{s_{1}}\left[\left(s_{2}-s\right)^{p-1}-\left(s_{1}-s\right)^{p-1}\right] g(w(s, \tau), s) d \mathbf{C}_{s}(\tau)\right| \\
& +\int_{s_{1}}^{s_{2}}\left(s_{2}-s\right)^{p-1}|f(w(s, \tau), s)| d s+\left|\int_{s_{1}}^{s_{2}}\left(s_{2}-s\right)^{p-1} g(w(s, \tau), s) d \mathbf{C}_{s}(\tau)\right| .
\end{aligned}
$$


By Lemma 2.1,

$$
\begin{aligned}
\leq & \frac{1}{\Gamma(p)} \int_{0}^{s_{1}}\left[\left(s_{2}-s\right)^{p-1}-\left(s_{1}-s\right)^{p-1}\right]|f(w(s, \tau), s)| d s \\
& +k(\tau) \int_{0}^{s_{1}}\left[\left(s_{2}-s\right)^{p-1}-\left(s_{1}-s\right)^{p-1}\right]|g(w(s, \tau), s)| d \mathbf{C}_{s}(\tau) \\
& +\int_{s_{1}}^{s_{2}}\left(s_{2}-s\right)^{p-1}|f(w(s, \tau), s)| d s+k(\tau) \int_{s_{1}}^{s_{2}}\left(s_{2}-s\right)^{p-1}|g(w(s, \tau), s)| d \mathbf{C}_{s}(\tau) .
\end{aligned}
$$

By a linear growth condition,

$$
\leq \frac{1}{\Gamma(p+1)}\left[\mathbf{L}(1+\|w(t, \tau)\|)(1+\mathbf{K}(\tau))\left(s_{2}^{p}-s_{1}^{p}\right)\right]
$$

Thus $\left|\mathbf{A}\left(w\left(s_{2}, \tau\right)\right)-\mathbf{A}\left(w\left(s_{1}, \tau\right)\right)\right| \rightarrow 0$ as $\left|s_{2}-s_{1}\right| \rightarrow 0$. So, $\mathbf{A}(w(s, \tau))$ is sample-continuous on $\mathbf{C}([-\tau, \mathbf{R}], \mathbf{R})$.

Theorem 3.1 (Existence and uniqueness) The FFDE (3.4) has a unique solution $w(t)$ in $[0,+\infty)$ if the coefficients $f(w(t), t)$ and $g(w(t), t)$ satisfy (I) and (II).

Proof Let $c>0$ be an arbitrarily given number, and let $\mathbf{A}(w(\xi))$ be a mapping defined by (3.4) on $w(\xi) \in C[t, t+c]$,

$$
\mathbf{A}(w(\xi))=w_{0}+\frac{1}{\Gamma(\alpha)} \int_{t}^{\xi}(t-s)^{p-1} f(w(s), s) d s+\frac{1}{\Gamma(\alpha)} \int_{t}^{\xi}(t-s)^{p-1} g(w(s), s) d C(s) . \quad \text { (3.9) }
$$

The proof of Lemma 3.2 implies that $\mathbf{A}(w(\xi)) \in \mathbf{C}[t, t+c]$ for $x(\xi) \in C[t, t+c]$.

For any $\xi \in[t, t+c]$, we have

$$
\begin{aligned}
\|\mathbf{A}(w(\xi, \tau))-\mathbf{A}(y(\xi, \tau))\|= & \max _{t \in[0, T]}|\mathbf{A}(w(\xi, \tau))-\mathbf{A}(y(\xi, \tau))| \\
\leq & \max _{t \in[0, T]} \frac{1}{\Gamma(p)} \mid \int_{t}^{\xi}(\xi-s)^{p-1}[f(w(s, \tau), s)-f(y(s, \tau), s)] d s \\
& +\int_{t}^{\xi}(\xi-s)^{p-1}[g(w(s, \tau), s)-g(y(s, \tau), s)] d \mathbf{C}_{s}(\tau) \mid .
\end{aligned}
$$

By Lemma 2.1,

$$
\begin{aligned}
\leq & \max _{t \in[0, T]} \frac{1}{\Gamma(p)} \int_{t}^{\xi}(\xi-s)^{p-1}|f(w(s, \tau), s)-f(y(s, \tau), s)| d s \\
& +\frac{k(\tau)}{\Gamma(p)} \int_{t}^{\xi}(\xi-s)^{p-1}|g(w(s, \tau), s)-g(y(s, \tau), s)| d \mathbf{C}_{s}(\tau) .
\end{aligned}
$$


By the Lipschitz condition,

$$
\begin{aligned}
& \left.\leq \frac{(1+k(\tau)) L}{\Gamma(p)} \max _{t \in[0, T]} \int_{t}^{\xi}(\xi-s)^{p-1} \mid(w(s, \tau), s)-y(s, \tau), s\right) \mid d s \\
& \leq \frac{(1+k(\tau)) L}{\Gamma(p)} \int_{t}^{\xi}(\xi-s)^{p-1} \sup _{t \leq s \leq t+c}|w((s, \tau), s)-y((s, \tau), s)| d s \\
& \leq \frac{(1+k(\tau)) L c^{p}}{\Gamma(p+1)}\|w(s, \tau)-y(s, \tau)\| .
\end{aligned}
$$

If we set $A(w(\xi, \tau))=w(t)$ for $\xi \in[t, t+c]$, then

$$
\|\mathbf{A}(w(\xi, \tau))-\mathbf{A}(y(\xi, \tau))\| \leq \beta(\tau)\|w((s, \tau), s)-y((s, \tau), s)\|,
$$

where $\beta(\tau)=\frac{(1+k(\tau)) L c^{p}}{\Gamma(p+1)}$. That is, $\mathbf{A}$ is a contraction mapping on $\mathbf{C}[t, t+c]$. Thus by the wellknown Banach fixed point theorem we have a unique fixed point $w(\xi, \tau) \mathbf{C}[t, t+c]$ which satisfies (3.9) in $[t, t+c]$.

Furthermore, $W(\xi, \tau)=\lim _{k \rightarrow 0} \mathbf{A}(W((\xi, k), \tau))$ where

$$
w(\xi, k), \tau)=\mathbf{A}(w(\xi, k-1), \tau)), \quad k=1,2, \ldots
$$

Suppose that $[0, c],[c, 2 c], \ldots,[k c, T]$ are the subsets of $[0, T]$ with $k c<T \leq(k+1) c$. The above process implies that the mapping A has a unique fixed point $W^{(i+1)}(\xi, \tau)$ with $W^{(i+1)}(i c, \tau)=W^{(i)}(i c, \tau)$ on the interval $[(i-1) c, i c]$ for $i=1,2, \ldots, k+1$ and setting $(k+1) c=$ $T$. Define $W(\xi, \tau)$ on the interval $[0, T]$ by setting

$$
w(\xi, \tau)= \begin{cases}w^{1}(\xi, \tau), & t \in[0, c], \\ \vdots & \\ x^{k}(\xi, \tau), & t \in[(k-1) c, k c], \\ x^{k+1}(\xi, \tau), & t \in[k c, T] .\end{cases}
$$

It is easy to see that $W(\xi, \tau)$ is the unique fixed point of $\mathbf{A}$ defined by (3.8) in $C[0, T]$. In addition, $W(\xi, \tau)=\lim _{k \rightarrow \infty} \mathbf{A}(W((\xi, k), \tau))$ where

$$
W((\xi, k), \tau)=\mathbf{A}(W(\xi, k-1), \tau)), \quad k=1,2, \ldots
$$

for any given $W((\xi, 0), \tau)=w(\xi) \in C[0, T]$. It follows from the arbitrariness of $T \geq 0$ that $W(\xi)$ is the unique solution of FFDE (3.4). Furthermore, since $W(\xi, \tau)$ is in $C[0, T], W(\xi)$ is sample-continuous. The theorem is proved.

\subsection{Stability theorem for FFDEs}

Regarding the fact that if a physical examination is repeated twice with the same conditions and how much measurements are conducted carefully, the initial imposed conditions will not be exactly the same. It is expected that the results of two physical examinations have a little difference with each other. This means that very small changes in initial conditions 
are expected to cause only small changes in the answer. In other words, the answer to the mathematical model is stable. In this section, the concept of stability for a FFDE in the sense of fuzzy measure is proposed. A sufficient condition will also be derived for a FFDE to be stable.

Corollary 3.1 Let $\mathbf{C}_{t}$ be a fuzzy process on a credibility space $(\Theta, \mathcal{P}, \mathbf{C r})$. Then there exists a fuzzy variable $\mathbf{K}$ in a way that $\mathbf{K}(\theta)$ is a Lipschitz constant of the sample path $\mathbf{C}_{t_{\theta}}$ for each $\theta$, and

$$
\lim _{x \rightarrow+\infty} \mathbf{C r}\{\theta \in \Theta \mid \mathbf{K}(\theta) \leq x\}=1
$$

Proof Define

$$
\mathbf{K}(\theta)=\sup _{0<t<s} \frac{\left|\mathbf{C}_{t_{\theta}}-\mathbf{C}_{s_{\theta}}\right|}{t-s}
$$

for each sample path $\mathbf{C}_{t_{\theta}}$. Then $\mathbf{K}$ is a fuzzy variable on $(\Theta, \mathcal{P}, \mathbf{C r})$, and

$$
\left|\mathbf{C}_{t_{\theta}}-\mathbf{C}_{s_{\theta}}\right| \leq \mathbf{K}(\theta)|t-s|
$$

for any $t_{1}, t_{2} \in \mathbf{R}^{+}$. That is, $\mathbf{K}(\theta)$ is a Lipschitz constant of the sample path $\mathbf{C}_{t_{\theta}}$. Given $x \in \mathbf{R}$, if

$$
\left|\frac{d \mathbf{C}_{t}}{d t}(\theta)\right| \leq x \quad \forall t>0
$$

then we have

$$
\left|\mathbf{C}_{t_{\theta}}-\mathbf{C}_{s_{\theta}}\right| \leq \int_{t}^{s}\left|\frac{d \mathbf{C}_{t}}{d t}(\theta)\right| d t \leq x(s-t)
$$

for any $t_{1}, t_{2} \in \mathbf{R}^{+}$, which means that

$$
\sup _{0<t<s} \frac{\left|\mathbf{C}_{t_{\theta}}-\mathbf{C}_{s_{\theta}}\right|}{s-t} \leq x .
$$

Thus we obtain

$$
\begin{aligned}
& \mathbf{C r}\{\theta \in \Theta \mid \mathbf{K}(\theta) \leq x\}=\mathbf{C r}\left\{\sup \frac{\mathbf{C}_{s}-\mathbf{C}_{t}}{s-t} \leq x\right\}, \\
& \mathbf{C r}\left\{\left|\frac{d \mathbf{C}_{t}}{d t}\right| \leq x, \forall t>0\right\} \leq 2 \phi(x)-1,
\end{aligned}
$$

where $\phi$ is the credibility distribution of standard normal fuzzy variable $\mathbf{N}(0,1)$. Since $\phi(x) \rightarrow 1$ as $x \rightarrow+\infty$, we have

$$
\lim _{x \rightarrow+\infty} \mathbf{C r}\{\theta \in \Theta \mid \mathbf{K}(\theta) \leq x\}=1 .
$$


Corollary 3.2 Let $\mathbf{C}_{t}$ be a fuzzy process on a credibility space $(\Theta, \mathcal{P}, \mathbf{C r})$, and $K(\theta)$ be the least Lipschitz constant of the sample path $\mathbf{C}_{t_{\theta}}$. Then

$$
\operatorname{Cr}\{\mathbf{K}<+\infty\}=1 .
$$

Proof We will prove this by a contradiction method. Assume that $\mathbf{C r}\{\mathbf{K}<+\infty\}<1$. Then there exists a positive number $\zeta>0$ such that $\mathbf{C r}\{\mathbf{K}<+\infty\} \leq 1-\zeta$, so we have $\mathbf{C r}\{\mathbf{K} \leq$ $x\} \leq 1-\zeta$ for any $x \in \mathbf{R}$. It means that

$$
\lim _{x \rightarrow+\infty} \mathbf{C r}\{\theta \in \Theta \mid \mathbf{K}(\theta) \leq x\} \leq 1-\zeta
$$

which contradicts

$$
\lim _{x \rightarrow+\infty} \mathbf{C r}\{\theta \in \Theta \mid \mathbf{K}(\theta) \leq x\}=1
$$

Definition 3.3 Let $w_{t}$ and $y_{t}$ be two solutions of the FFDE

$$
\left\{\begin{array}{l}
{ }^{C} D^{\alpha} w(t)=f(w(t), t)+g(w(t), t) \dot{C}(t), \\
w(t)=w_{t} .
\end{array}\right.
$$

Then the FFDE (3.10) is said to be stable if for any given number $\xi>0$, there exists a real number $\delta$ such that

$$
\mathbf{C r}\{|w(t)-y(t)|>\varepsilon\} \leq \varepsilon,
$$

or

$$
\lim _{\left|w_{0}-y_{0}\right| \rightarrow 0} \mathbf{C r}\{|w(t)-y(t)|>\varepsilon\}=0, \quad \forall t>0,
$$

holds for any $t \geq 0$ provided $\left|w_{0}-y_{0}\right| \leq \delta$.

Theorem 3.2 The FFDE (3.10) is stable if the coefficients $f(w, t)$ and $g(w, t)$ satisfy (I) and (II).

Proof Assume that $w(t)$ and $y(t)$ are two solutions of the FFDE (3.10) with different initial values $w(0)$ and $y(0)$, respectively. Then

$$
\begin{aligned}
& w(t)=w_{0}+\frac{1}{\Gamma(\alpha)} \int_{0}^{t}(t-s)^{\alpha-1} f(w(s), s) d s+\frac{1}{\Gamma(\alpha)} \int_{0}^{t}(t-s)^{\alpha-1} g(w(s), s) d C(s), \\
& y(t)=y_{0}+\frac{1}{\Gamma(\alpha)} \int_{0}^{t}(t-s)^{\alpha-1} f(y(s), s) d s+\frac{1}{\Gamma(\alpha)} \int_{0}^{t}(t-s)^{\alpha-1} g(y(s), s) d C(s) .
\end{aligned}
$$


For a Lipschitz-continuous sample path $\mathbf{C}_{t_{\theta}}$, we have

$$
\begin{aligned}
& |w(t, \theta)-y(t, \theta)| \\
& \leq|w(0)-y(0)|+\frac{1}{\Gamma(p)} \int_{0}^{t}(t-s)^{p-1}|f(w(s, \theta))-f(y(s, \theta))| d s \\
& \quad+\frac{1}{\Gamma(p)} \int_{0}^{t}(t-s)^{p-1}|g(w(s, \theta))-g(y(s, \theta))|\left|d \mathbf{C}_{s}\right| \\
& \leq|w(0)-y(0)|+\frac{1}{\Gamma(p)} \int_{0}^{t}(t-s)^{p-1} \mathbf{L}(s)|w(s, \theta)-y(s, \theta)| d s \\
& \quad+\frac{1}{\Gamma(p)} \int_{0}^{t}(t-s)^{p-1} \mathbf{L}(s) \mathbf{K}(\theta)|w(s, \theta)-y(s, \theta)| d s \\
& =|w(0)-y(0)|+\frac{1}{\Gamma(p)} \int_{0}^{t}(t-s)^{p-1} \mathbf{L}(s)(1+\mathbf{K}(\theta))|w(s, \theta)-y(s, \theta)| d s .
\end{aligned}
$$

Thus we have

$$
|w(t, \theta)-y(t, \theta)| \leq|w(0)-y(0)|+\exp (1+\mathbf{K}(\theta)) \frac{c^{p}}{\Gamma(p+1)} \int_{0}^{\infty} \mathbf{L}(s) d s,
$$

by the Grownwall equality.

For any given $\varepsilon>0$, there exists a real number $\mathbf{H}$ such that $\mathbf{C r}\{\theta \mid \mathbf{K}(\theta) \leq \mathbf{H}\} \geq 1-\varepsilon$, by Corollary 3.1 take

$$
\delta=\exp \left(-(1+\mathbf{H}) \int_{\tau}^{\infty} \mathbf{L}(s) d s\right) \varepsilon
$$

Then $|w(t, \theta)-y(t, \theta)| \leq \varepsilon$ for any positive real number $t$, provided that $|x(0)-y(0)| \leq \delta$ and $\mathbf{K}(\theta) \leq \mathbf{H}$. It means $\mathbf{C r}\{|w(t)-y(t)|>\varepsilon\}<\varepsilon$ for any $t \leq 0$ as long as $|w(0)-y(0)| \leq \delta$. In other words, $\lim _{\left|w_{0}-y_{0}\right| \rightarrow 0} \mathbf{C r}\{|x(t)-y(t)|>\varepsilon\}=0$ so the FFDE is stable.

\section{Conclusion}

In this paper, weaker conditions are provided in order to guarantee the existence and uniqueness of the solution to the fuzzy fractional deferential equations driven by Liu's process, which makes it possible for more functions to be verified in such conditions. Furthermore, stability can also be investigated by considering such conditions.

Competing interests

The authors declare that they have no competing interests.

Authors' contributions

All authors read and approved the final manuscript.

\section{Publisher's Note}

Springer Nature remains neutral with regard to jurisdictional claims in published maps and institutional affiliations.

Received: 24 March 2017 Accepted: 24 July 2017 Published online: 16 August 2017

\section{References}

1. Miller, KS, Ross, B: An Introduction to the Fractional Calculus and Differential Equations. Wiley, New York (1993)

2. Oldham, KB, Spanier, J: The Fractional Calculus. Academic Press, New York (1974) 
3. Samko, SG, Kilbas, AA, Marichev, OI: Fractional Integrals and Derivatives, Theory and Applications. Gordon \& Breach, New York (1993)

4. Lakshmikantham, V, Leela, S, Vasundhara, D: Theory of Fractional Dynamic Systems. Cambridge Academic Publishers (2009)

5. Liu, W, Jiang, W: Uniform stability of fractional neutral systems: a Lyapunov Krasovskii functional approach. Adv. Differ. Equ. 2013, article 379 (2013)

6. Ahmood, WA, Kiliman, A: On some applications of the space-time fractional derivative. Adv. Differ. Equ. 2016, article $288(2016)$

7. Hale, J: Theory of Functional Differential Equations. Springer, New York (1997)

8. Ahmad, B, Nieto, JJ: Existence results for a coupled system of nonlinear fractional differential equations with three-point boundary conditions. Comput. Math. Appl. 58, 1838-1843 (2009)

9. Ahmad, B, Ntouyas, SK, Agarwal, RP, Alsaedi, A: On fractional differential equations and inclusions with nonlocal and average-valued (integral) boundary conditions. Adv. Differ. Equ. 2016, article 80 (2016)

10. Ding, Z, Ma, M, Kandel, A: Existence of the solutions of fuzzy differential equations with parameters. Inf. Sci. 99(1), 1205-1217 (1999)

11. Kilbas, AA, Srivastava, HM, Trujillo, Jj: Theory and Applications of Fractional Differential Equations. Elsevier Amsterdam (2006)

12. Lakshmikantham, V, Vatsala, AS: Basic theory of fractional differential equations. Nonlinear Anal. 69, 2677-2682 (2008)

13. Podlubny, I: Fractional Differential Equation. Academic Press, San Diego (1999)

14. Sakulrang, S, Moore, EJ, Sungnul, S, de Gaetano, A: A fractional differential equation model for continuous glucose monitoring data. Adv. Differ. Equ. 2017, article 150 (2017)

15. Bao, $\mathrm{H}, \mathrm{CaO}$, J: Existence of solutions for fractional stochastic impulsive neutral functional differential equations with infinite delay. Adv. Differ. Equ. 2017, article 66 (2017)

16. Ciotir, I, Răşcanu, A: Viability for differential equations driven by fractional Brownian motion. J. Differ. Equ. 247, 1505-1528 (2009)

17. Cui, J, Yan, L: Existence result for fractional neutral stochastic integro-differential equations with infinite delay. J. Phys. A, Math. Theor. 44(33), 335201 (2011)

18. El-Borai, MM, El-Said El-Nadi, K, Fouad, HA: On some fractional stochastic delay differential equations. Comput. Math. Appl. 59, 1165-1170 (2010)

19. Lu, Y, Yao, Z, Zhu, Q, Yao, Y, Zhou, H: Comparison principle and stability for a class of stochastic fractional differential equations. Adv. Differ. Equ. 2014, article 221 (2014)

20. Merdan, M, Bekiryazici, Z, Kesemen, T, Khaniyev, T: Comparison of stochastic and random models for bacterial resistance. Adv. Differ. Equ. 2017, article 133 (2017)

21. Pei, $B, X u, Y:$ On the non-Lipschitz stochastic differential equations driven by fractional Brownian motion. Adv. Differ. Equ. 2016, article 194 (2016)

22. Xia, D, Yan, L: Some properties of the solution to fractional heat equation with a fractional Brownian noise. Adv. Differ Equ. 2017, article 107 (2017)

23. Zhang, X, Agarwal, P, Liu, Z, Peng, H, You, F, Zhu, Y: Existence and uniqueness of solutions for stochastic differential equations of fractional-order $q>1$ with finite delays. Adv. Differ. Equ. 2017, article 123 (2017)

24. Zadeh, LA: Fuzzy sets. Inf. Control 8(1), 338-353 (1965)

25. Ahmadian, A, Suleiman, M, Salahshour, S, Baleanu, D: A Jacobi operational matrix for solving a fuzzy linear fractional differential equation. Adv. Differ. Equ. 2013, article 104 (2013)

26. Friedman, M, Ming, M, Kandel, A: Numerical procedures for solving fuzzy differential and integral equations. In: International Conference Fuzzy Logic and Applications, Israel, pp. 18-21 (1997)

27. Kaleva, O: Fuzzy differential equation. Fuzzy Sets Syst. 24, 301-317 (1987)

28. Salahshour, S, Allahviranloo, T, Abbasbandy, S, Baleanu, D: Existence and uniqueness results for fractional differential equations with uncertainty. Adv. Differ. Equ. 2012, article 112 (2012)

29. Liu, B: Uncertainty Theory. Springer, Berlin (2004)

30. Li, X, Liu, B: A sufficient and necessary condition for credibility measures. Int. J. Uncertain. Fuzziness Knowl.-Based Syst. 14(5), 527-535 (2004)

31. Liu, B: A survey of credibility theory. Fuzzy Optim. Decis. Mak. 5(4), 387-408 (2006)

32. Liu, B: Uncertainty Theory, 2nd edn. Springer, Berlin (2007)

33. Dai, W: Lipschitz continuity of Liu process. In: Proceedings of the Eighth International Conference on Information and Management Science, China, pp. 756-760 (2009)

34. Gao, J: Credibilistic option pricing. J. Uncertain Syst. 2(4), 243-247 (2008)

35. Liu, B: Fuzzy process, hybrid process and uncertain process. J. Uncertain Syst. 2(1), 3-16 (2008)

36. Liu, B, Liu, Y: Expected value of fuzzy variable and fuzzy expected value models. IEEE Trans. Fuzzy Syst. 10(4), 445-450 (2002)

37. Liu, B: Some research problems in uncertainty theory. J. Uncertain Syst. 3(1), 3-10 (2009)

38. Peng, J: A general stock model for fuzzy markets. J. Uncertain Syst. 2(4), 248-254 (2008)

39. Liu, H, Fei, W: Neutral uncertain delay differential equations. Information 16(2), 1225-1232 (2013)

40. Qin, Z, Gao, X: Fractional Liu process with application to finance. Math. Comput. Model. 50(9), 1538-1543 (2009)

41. Yao, K: A type of nonlinear uncertain differential equations with analytic solution. J. Uncertain. Anal. Appl. 8(1), 10-21 (2013)

42. You, C: Multi-dimensional Liu process, differential and integral. In: Proceedings of the First Intelligent Computing Conference, China, pp. 153-158 (2007)

43. Zhu, Y: Existence and uniqueness of the solution to uncertain fractional differential equation. J. Uncertain. Anal. Appl. 3(5), 1-11 (2015)

44. Qin, Z, Li, X: Option pricing formula for fuzzy financial market. J. Uncertain Syst. 2(1), 17-21 (2008)

45. You, C, Wang, W, Huo, H: Existence and uniqueness theorems for fuzzy differential equations. J. Uncertain Syst. 7(4), 303-315 (2013)

46. Fei, W: Uniqueness of solutions to fuzzy differential equations driven by Lius process with non-Lipschitz coefficients In: International Conference on Fuzzy and Knowledge Discovery, pp. 565-569 (2009)

47. Chen, X, Qin, X: A new existence and uniqueness theorem for fuzzy differential equations. Int. J. Fuzzy Syst. 13(2), 148-151 (2013) 